\title{
Evaluation Study of Heart Valve Replacement for Patients Aged from 10 to 80 Years at Al-Nasiriyah Heart Center/Iraq
}

\author{
${ }^{1}$ Hussein Togun, ${ }^{2}$ Munaf Ahmed, ${ }^{1}$ Lina Rabee and ${ }^{1}$ Farah Adel \\ ${ }^{1}$ Department of Biomedical Engineering, University of Thi-Qar, 64001 Nassiriya, Iraq \\ ${ }^{2}$ Al-Nasiriyah Heart Center Thi-Qar, 64001 Nassiriya, Iraq
}

\begin{abstract}
The problems in the aortic and Mitral Valves (MV) are represented most often heart diseases. Heart valve replacement for patients aged from 10-80 years at Al-Nasiriya Heart Center studied in this study. The 87 cases, reporting predication of patients who underwent surgery for heart valve, information on the patients, heart valve diseases, causes valve diseases, valve diseases diagnosed, valve diseases treated and types of valve replacement. The results adopted 87 cases, mitral valve was the most valve has been replaced in 46 cases $(53 \%)$ while aortic valve replaced in 27 cases $(31 \%)$ and the double valve replaced in 14 cases $(16 \%)$. Number of cases in 2014 was $28(32 \%)$ in 2015 was $26(30 \%)$ in 2016 was $8(9 \%)$ and in 2017 was $25(29 \%)$. The higher number of patients who underwent heart valve replacement was 27 for age between $40-50$ years because at this age the valve will be more active and due to lack of attention and health care to prevent and avoid heart valve causes that will lead to damage and diseases, thus, at the end the damaged heart valve must be replaced. While the lowest number was 2 for aged between 70-80 years. The total center mortality from 2014-2017 was just two cases in 26th May. About 2014 patient underwent to MVR surgery at age about 10 years old died because of Deep Vein Thrombosis (DVT). In 17th Feb. 2015 patient underwent to AVR surgery at age 41 years old died because of Cerebrovascular Accident (CVA).
\end{abstract}

Key words: Mitral valve, aortic valve, surgical heart valve replacement, pulmonary valve, heart diseases, mortality

\section{INTRODUCTION}

Heart diseases are the primary cause of death in many developed countries. The problems in the Aortic (AV) and Mitral Valves (MV) are represented most often heart diseases. Generally, there are two types of cardiac valves.

\section{The semilunar valves}

The Aortic Valve (AV): Lies behind the left half of sternum opposite the 3 rd intercostals space.

The Pulmonary Valve (PV): Lies behind the medial end of the third left costal cartilage and the adjoining part of the sternum.

\section{The atrioventricular valves}

The tricuspid valve (TV): Lies behind the right half of the sternum opposite the 4th intercostal space.

The Mitral Valve (MV): Lies behind the left half of the sternum opposite the 4th costal cartilage (Snell, 2000).
In 1952, the first mechanical prosthetic valve has been medically used by Dr. Charles Hufnagel. It inserted a prosthetic valve consisting of a nylon ball and poly methyl methacrylate tube into the descending aorta to correct the incompetence of aortic valve. Some of those prostheses worked up to 18 years but this happened in 1953, after the heart-lung machine had introduced by Gibbon where it employed through open-heart surgery which in turn allow to an open-heart surgery to perform under direct vision. In the late, 1950 's, the first surgery for stenosis of calcific aortic where the calcium removed from the valves. But it was not a successful method, since, the calcium returned after a few years and a lot of valves after decalcification they were destroyed. So, after that the right function of these valves could never restored. The artificial heart valves made of Teflon (Bahnson valves) were the first that copied the heart valves of human body. These artificial heart valves encapsulated by a thin sheet of fibrous tissue (in blood stream) after the fibrin and platelets had been deposited. These pocked valves became insufficient due to their tendency to shrink down after 1-2 years, so that, the edge of these valves turned in due to the deposition of much amount of calcium on the

Corresponding Author: Hussein Toguna, Department of Biomedical Engineering, University of Thi-Qar, 64001 Nassiriya, Iraq 
Teflon leaflets, so, it made the leaflets ruptured between 1 and 2 years. Hufnagel used silicone rubber to cover the Teflon valves to prevent deposition of calcium and prevent them from rupturing but nevertheless Teflon valves also ruptured and all these valves had to be exchanged. In 1960 was the beginning of the modern era of the replacement of prosthetic valve by Dwight Harken by inserted into the aortic orifice below the coronary ostia a double cage-ball valve following the diseased cusps had been excised. Some of the patients who had this surgery stayed alive after more than 15 years. Also, in 1960s, Lowell Edwards and surgeon Albert Star made a prosthetic heart valve consisted a ball made from silicon and one cage made from poly methyl methacrylate they used one cage to make it a simple valve and also by introducing sewing ring, it made the valve easier to suture in place. It became available in commercial in 1962. It was a reliable mechanical valve and patients after 19 years still with functioning ball valves. But with the ball in the center of the bloodstream this valve was central occluding valve and causing a certain obstruction bloodstream (Morse et al., 2012).

A low-profile disc valve introduced in 1965 by Donald Shiley and Kay, this valve did not extend into the narrow area of the ridge of supravalvular. They tried to make these valves from pericardium of the patient but within a few years these valves ruptured and calcified. Surgeon Juro Wada developed a tilting disc valve and in as compare to ball valves a tilting disc valve functioned better (Morse et al., 2012).

On January 16, 1969 was the first introduced of developed tilting disc valve with a free floating Delrin disc in a patient. It developed by Viking O. Bjork with Shiley of Irvine, Calif. They used Delrin instead of Teflon, since, Delrinmore resistant to wear. However, In 1971 it was changed to a disc made of a material could not be worn out and it harmless to the blood (carbon pyrolite). The reason for changing Delrin was its tendency to absorb moisture and this resulted few changes in configuration it could swell and make them to become stuck in the ring (DeWall et al., 2000).

\section{MATERIALS AND METHODS}

Heart valve disease: The heart function is to circulate the blood to the lung in closed circuit where blood is oxygenated and out to the body and oxygen provides cellular metabolism's fuel. This task accomplished by pumping blood from the body to the lung by the right heart system once the blood oxygenated in the lungs, blood should be returned to the left heart, then pumped out to the body. When diseases affecting the heart valve will result in obstruction to forward flow (stenosis) or will cause reversal of flow across an incompetent valve (regurgitation) (Iaizzo, 2009).

Aortic stenosis: In Western world and after hypertension and coronary artery disease, Aortic Stenosis (AS) is the most common form of cardiovascular disease. It is caused usually by either degenerative calcification of tri leaflet or caused by progressive stenosis of congenital valve. Development of aortic stenosis occurs by progressively calcification of leaflets with restriction of leaflet opening over time. For the development of degenerative calcific AS, risks factors are similar to those for the development of vascular atherosclerosis. Risks factor including diabetes, smoking, hypertension and elevated levels of low-density lipoprotein cholesterol and lipoprotein. Patients suffering from valvular AS, stenosis severity increases gradually over many years. The left ventricle adopt to the obstruction by increasing the thickness of the wall while maintaining normal LV chamber size (concentric hypertrophy). When dysfunction of LV systolic is present, it often improve after Aortic Valve Replacement (AVR). But function of $\mathrm{LV}$ is not improve if myocardial contractile dysfunction is irreversible (Maganti et al., 2010).

Aortic regurgitation: Aortic regurgitation results from aortic leaflets abnormalities, the supporting structures in the aortic root and annulus or both. Rheumatic heart disease stays the most widespread cause of severe AR worldwide. However, diseases including the ascending aorta and aortic root became more frequent causes of $\mathrm{AR}$ in the Western hemisphere. The aortic cusps abnormalities that may be result in AR include congenital leaflet abnormalities, like bicuspid, unicuspid or quadricusps another congenital defects like subaortic membranes. Enlargement of the aortic root causes AR by annular dilatation, resulting in leaflet separation and loss of comptation. Bicuspid aortic valves are usually related with dilatation of the aortic root as congenital leaflet abnormality because of the aortic matrix abnormalities. It is important to be notice that chronic AR by itself may be lead to over time to progressive aortic root dilatation (Wang et al., 2007).

Mitral regurgitation: Mitral regurgitation may result from the valve leaflets disorders. The leading reas on of $M R$ is rheumatic heart disease and degenerative forms of MV disease (myxomatous disease and fibroelastic deficiency). Less popular conditions contain mitral annular calcification and congenital anomalies like cleft MVs. The second leading reason of $M R$ is functional $M R$ which 
(a)

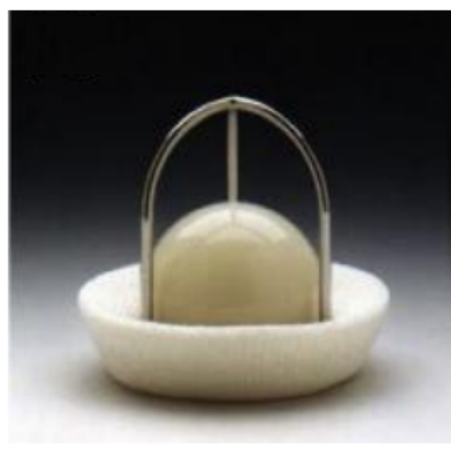

(b)

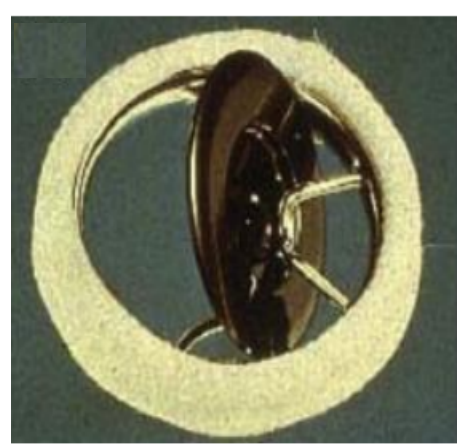

(c)

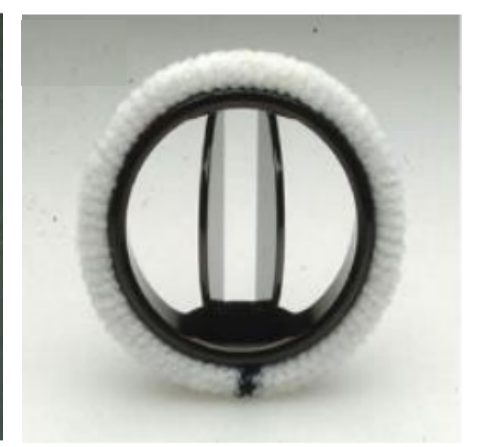

Fig. 1: Mechanical prosthetic heart valves: a) Starr-Edwards caged-ball valve; b) Bjork-Shiley tilting disk valve and c) St. Jude medical bileaflet tilting disk heart valve

occurs from dilatation of the MV annulus or from myocardial infarction. Particularly, infarctions containing the posteromedial and the inferolateral papillary muscle will produce the mitral leaflets tethering that will prohibit normal cooptation and leading to functional MR even though the valve leaflets are normal. Patients who have developed acute severe MR usually exist with symptomatic heart failure because the ill ventricles prepared to be accepted the sudden increase in volume load. The left atrium also will enlarge, so will allow the regurgitant volume accommodation at a lower pressure (Maganti et al., 2010).

Mitral stenosis: Rheumatic fever is the most common reason of MS and isolated MS in women is twice than in men (Wang et al., 2007). Another causes of MS are very uncommon and contain congenital anomalies, prior exposure to chest radiation, mucopolysaccharidosis and left atrial myxoma. The MS first symptoms are commonly exertional dyspnea and fatigue. There are patients may be also present with pulmonaryedema, atrialfibrillation.

Types of valve replacement: Generally, there are two generic types of Cardiac valvular substitutes and can be classified to mechanical valves and biological tissue valves. The first implantation of the valve in a beating heart was done by Charles Hufnagel in 1952 which it is consisting of a nylon ball and poly methyl methacrylate tube. Though, it was unsuccessful operation but it was fascinating. And also it's inspired the possibility of prosthetic valves to other cardiac surgeons. Later in 1953, Gibbon used the heart lung machine where developed it and occupied through open-heart surgery by in turn allow to the evolution of prosthetic heart valves to happen. In 1960 in America, a mitral valve replacement (consisted a ball made from silicon and cage made from poly methyl methacrylate) invented by engineering Lowell Edwards and implanted by surgeon Albert Star in a human (El Oakley et al., 2008; Rahimtoola, 2010 ).
Mechanical prosthetic heart valves (Fig. 1) are consist of non-physiologic biomaterials that involve rigid, mobile occludes in a metallic cage (cobalt-chrome or titanium alloy), like in the Bjork-shiley, Medtronic-Hall and Omniscience valves or two carbon hemidisks in a carbon housing as in the St. Jude medical, Carbomedics CPHVm and the medical carbon research institute or On-X prostheses. Recently, all mechanical valve occludes have been fabricate from pyrolytic carbon. This pyrolytic carbon has high strength, fatigue and wears resistance and exceptional biocompatibility including thrombo resistance. Patients those receiving mechanical valves should be treated with lifelong anticoagulation in order to reduce the risk of thrombosis and thromboembolic events. Figure 1 showed that the tissues valves resemble natural valves more than do mechanical prostheses. The term "bioprostheses" describes a special type of tissue valve that consists from three cusps of tissue that drive from animals the most widely used either a porcine (pig) aortic valve or bovine (cow) pericardium each of them treated with glutaraldeyde. Gultaraldehyde fixation preserves the tissue and decreases it's (it is already low) immunological reactivity and will kill the cell within the valve tissue. No immunosuppression is in general has been used for these xenografts as is required for whole organ transplants (e.g., kidney, liver or heart). Since, these valves no longer contain viable cells, so, the cusps themselves cannot respond to injury like normal tissue can do. Bioprosthetic valve cusps are mounted on a metal or plastic stent with three posts (or strust) to stimulate the geometry of a native semilunar valve. As with mechanical valves, the base ring has been covered by a Dacron or Teflon covered sewing cuff to facilitate surgical implantation and healing. Commonly used bioprosthetic valves are the Hancock porcine, Carpentier Edwards porcine and Carpentier-Edwards pericardial tissue valves. Also, occasionally tissue valves drive from human cadaveric aortic or pulmonary valves with or without the associated vascular conduit (called allografts or homografts) 


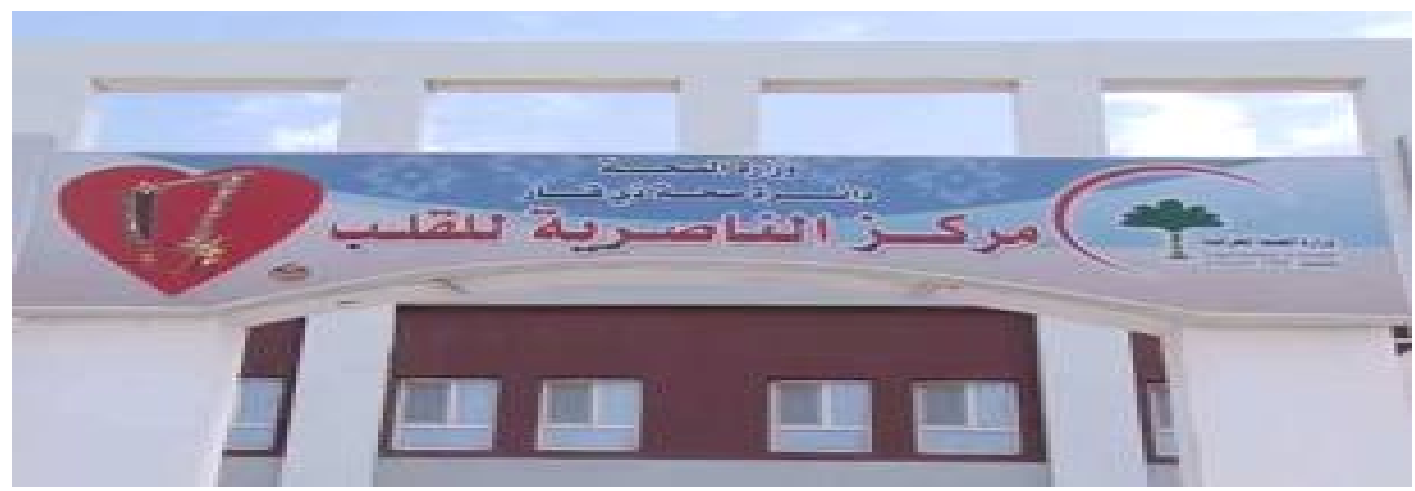

Fig. 2: Nasiriyah Heart Center

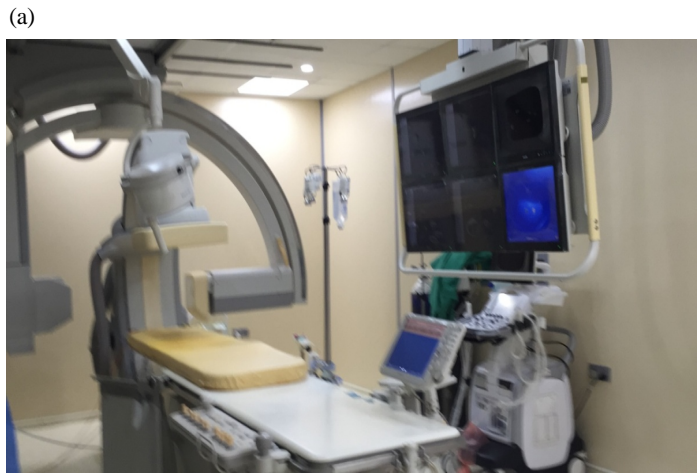

(b)

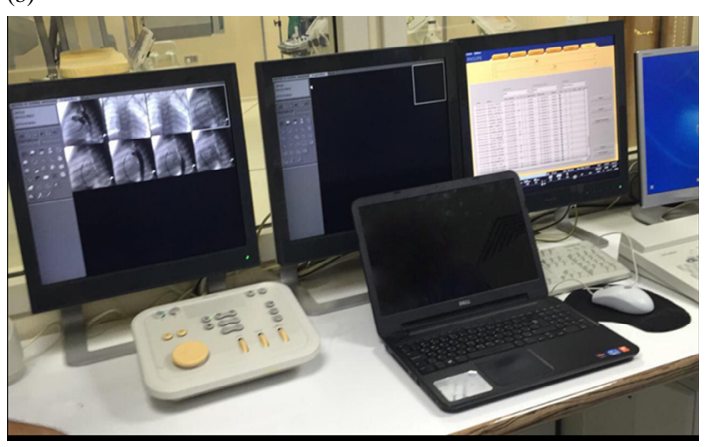

Fig. 3a): The catheterization January 3, 2019 operation room and b) The control panel of the catheterization room

(O'Brien et al., 2001). The aim of this study is to analyze and assessment morbidity and mortality rate that associated with heart valve replacement for patients aged from $10-80$ years old.

Overview about Nasiryah Heart Center: Nasiriyah Heart Center (Fig. 2) is considered one of the important specialized centers in Thi-Qar which specializes in the diagnosis and treatment of heart disease. The center has been opened on 10, July, 2007, It employs a specialized staff of doctors, engineers and specialized medical and technical staff. The establishment of this center in Thi-Qar is a unique experience to be the first center in South of Iraq in this rare specialty and is an important step in the transfer of strategic projects from the capital to the rest of the provinces to provide medical and therapeutic services for heart patients in the Thi-Qar Province in addition to the other provinces.

The sections of center: As shown in Fig. 3a, b the center includes the following sections.

Consulting section: This study provides consultation service to the patient by doctors. Contains an ECG device and echocardiography device.

Children's consultation: The consultation service is provided for children in particular. The room also contains both devices (ECG and EKG). Cardiac stress test room and ECG recording (Holter).

Department of catheterization: There are some cases that a doctor turns from a consultant to a catheterization room as shown in Fig. 3a, b. There are two types of catheterizations.

Diagnostic catheterization: This is a condition in which the doctor performs the catheterization to diagnose the condition.

Therapy catheterization: The doctor then performs the surgery for the patient as the implantation of the pacemaker, etc.

Department of X-ray: As shown in Fig. 4 the Department of Radiology at the Nasiriyah Heart Center is one of the most important departments in the center including different devices. Where it has the effectiveness and responsibility in diagnosing the most heart diseases, including blockage of the arteries and facilitate the operation of cardiac catheterization. 


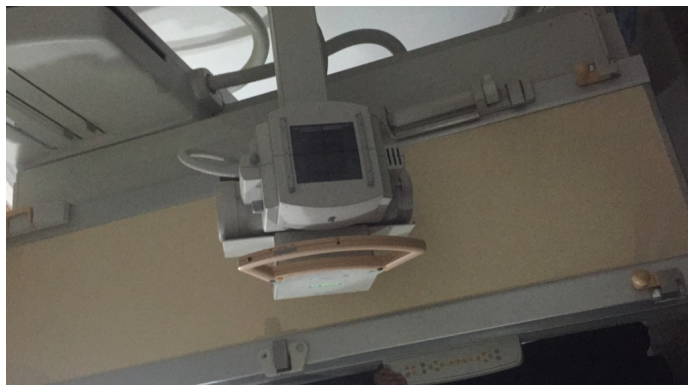

Fig. 4 : X-ray diagnostic room

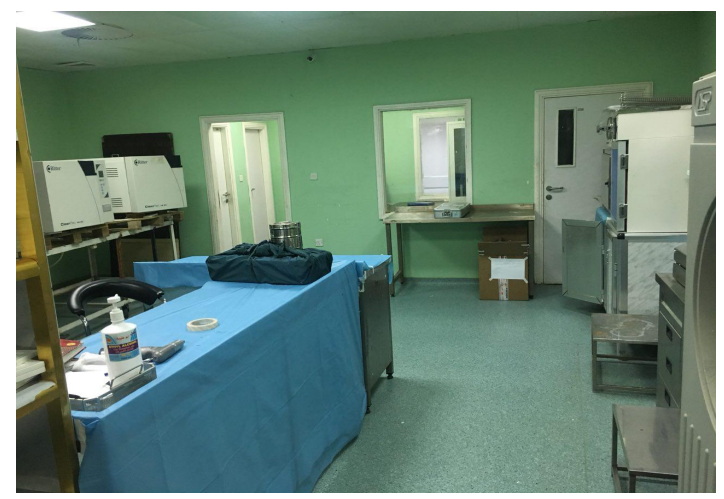

Fig. 5: Sterilization room

Maintenance department: General Maintenance Department: this study provides all the needs of the center in terms of maintenance and others and includes a staff of engineers from the Department of Electricity, Mechanics and Civil as well as includes a number of technicians and crafts of the same sections. The Department of Medical Maintenance provides the Center's needs as well as maintenance and medical equipment including a staff of engineers for medical devices and biomedical engineering.

Department of congenital heart defects: Contributes to the addition of a specialized service to the center for the treatment of this segment of patients. The center also includes completely separate men's and women's lounges and children's lounges as well. In addition to the administrative departments and the educational section which includes a lecture hall equipped with all educational means.

\section{Department of surgery}

Sterilization room: Contains a number of special devices for disinfection of instruments and surgical materials which include plasma sterilizer device, EO sterilizer device oven sterilizer and autoclave show in Fig. 5.

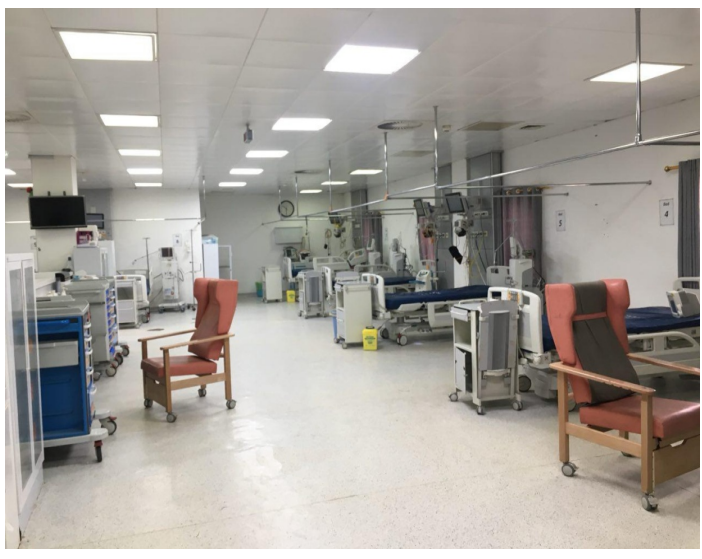

Fig. 6: Intensive Care Unit (ICU)

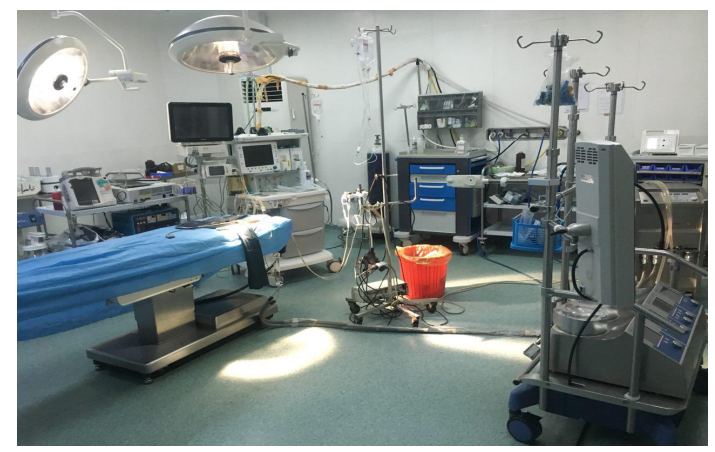

Fig. 7: Operation room

Intensive Care Unit (ICU): As shown in Fig. 6 is the surgical resuscitation room is where the patient transferred to it after completion of surgery. Laboratory to measure the proportion of blood gases.

Operation rooms: Where surgical operations are performed in a sterile environment, Fig. 7. The surgeries performed by the doctors are open heart surgery, repair of congenital heart defects and repair or replacement diseased heart valves surgeries, etc. It's contain different devices overhead surgical lights, operating table, monitors, suction unit medical device, electrosurgery unit, anesthesia machine and heart lung machine.

Heart-lung machine: As shown in Fig. 8, operating on the human heart poses problems which inhibited surgery on the heart until the early 1950's. Manipulation of the heart and opening of its cavitie's interferes with its function and its ability to sustain the circulation. The heart-lung machine is a system which takes over the function of the heart and the lungs with sufficient safety to maintain life while the heart is stopped or opened to allow surgery on the coronary arteries or the heart valves 


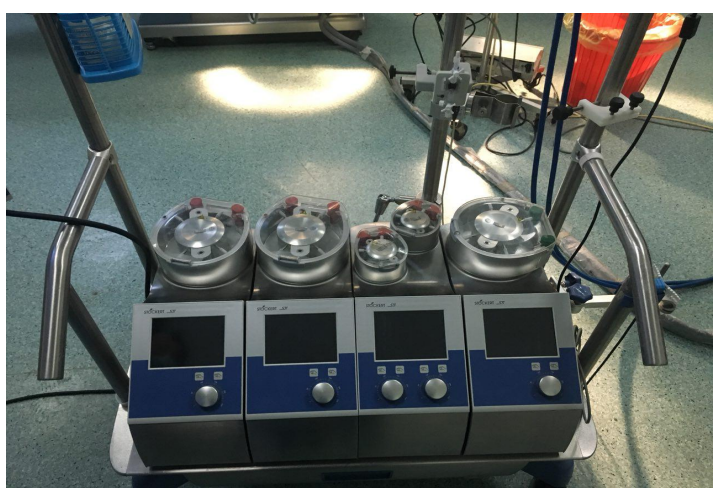

Fig. 8: Heart-lung machine

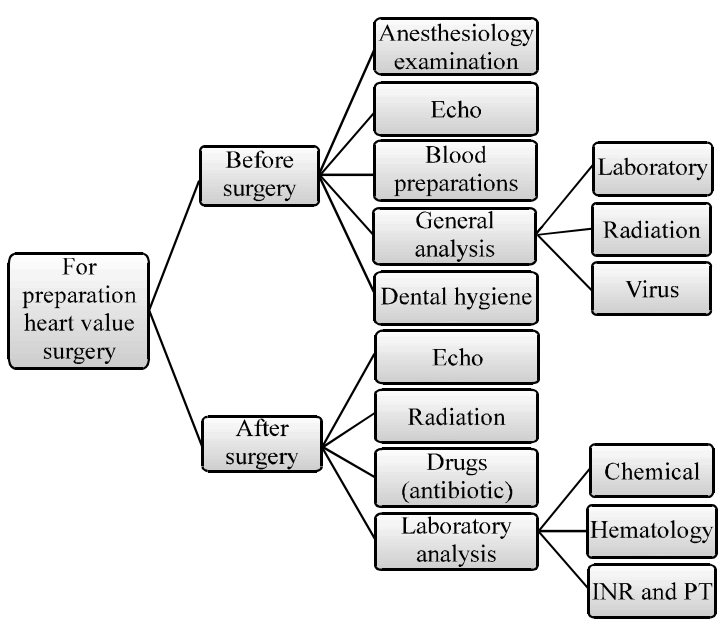

Fig. 9: Preparation before and after heart valve surgery flowchart

or to allow repair of congenital abnormalities. At the Nasiriyah Heart center the S5 heart-lung machine is used. Sorin's 5th-generation modular heart-lung machine has set new standards for functionality, flexibility and quality. Designed for many years of demanding performance, the advanced design allows for:

- Better integration with the perfusion circuit

- More configuration options to accommodate a greater assortment of procedures (including pediatric)

- Quick and easy exchange of individual components in the most challenging environments

- Unprecedented safety features some preparations required before and after heart valve surgery shown in Fig. 9

Some preparations required before and after heart valve surgery when visiting the Al-Nassiriyah Heart Center. Before the surgery, first, the patient need general

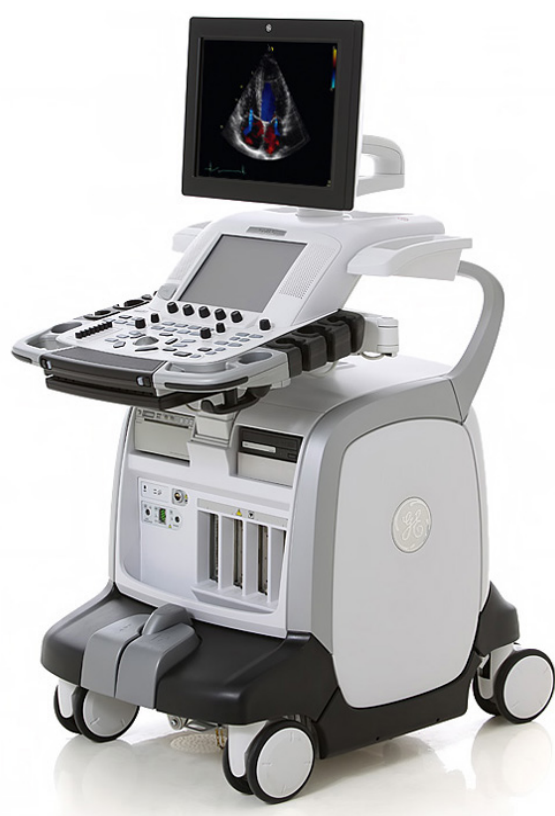

Fig. 10: Echocardiography device

analysis that include laboratory, radiation and virus analysis. Also, dental hygiene because teeth is always source for bacteria when they attack valves they can't get out from it because the valve has no blood supply, thus, even when we give antibiotic they can't reach to the valve, then the valve must be replaced. Anesthesiology examination include function of lung also laboratory, chemical and biology examination. blood preparation include fresh blood, donors who identical to the patients, or whole banked that mean the blood is kept (cold blood) in addition to blood product like plasma and platelet because in some cases we need just plasma or platelet not the blood.

In addition to other analytical devices that used for perform different analytical tests for patients, the device is used for diagnosis of heart diseases is echocardiogram device this device is used to make a sonogram to heart (used sound waves to produce images of heart) and depending on the information the doctor needs, patient may have one of the following kinds of echocardiograms which should be used before and after surgery:

- Transthoracic echocardiogram

- Transesophageal echocardiogram

- Doppler echocardiogram

- Stress echocardiogram

In Nasiriyah Heart Center the ECHO device that used is echocardiogram E9 from GE company, (Fig. 10). In 

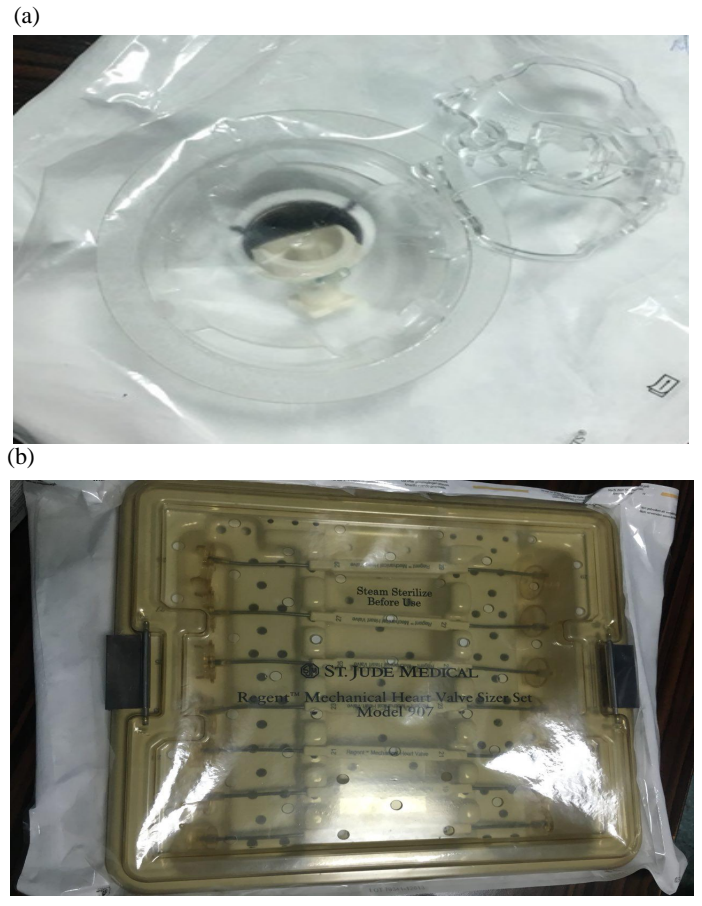

Fig. 11a): St-Jude mechanical heart valve and b) St. Jude mechanical heart valve measurement tools

addition to that there are some cases diagnostic catheterization performed and also diagnostic procedure performed by using CT scan and MRI. The treatment is performed by replacing the diseased valve or repairing it. The repair of diseased heart valves is performed for patients who are $<12$ years old because if the valve in these ages is replaced it's need another replacement surgery in the future, since, the diameter of valve changes as the patient continues to grow. Except in difficult cases that's need to replace the diseased valves.

For patients who are more than 12 years old a replacement surgery by mechanical or tissue valve is performed mechanical valve is made of mechanical parts which are tolerated well by the body and it's more efficient and remain for long period of time than bioproshetic valve (tissue valve). So, that, the tissue valve since it remains approximately for 10 years is used only for patients who are more than 75 years old. In Nasiriyah Heart Center only mechanical valves are used from ST Jude Medical company and from Sorin Group company.

The St. Jude heart valve as shown in Fig. 11a was the first bileaflet mechanical heart valve This bileaflet mechanical heart valve is designed and manufactured entirely from pyrolytic carbon. While the swing cuff made from double velour Dacron. Fig. 12b show St. Jude mechanical heart valve measurement.

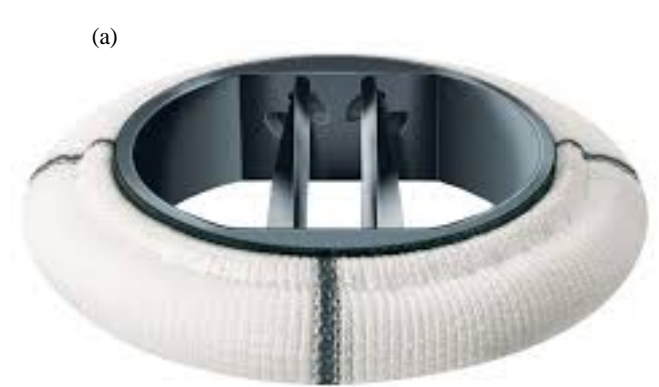

(b)

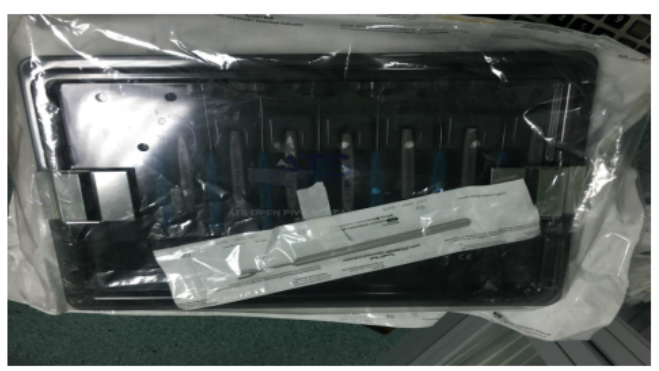

Fig. 12a): Sorin mechanical heart valve and b) Sorin mechanical heart valve measurement tools

In Fig. 12a, b they are show the sorin group mechanical heart valve that's also made from pyrolytic carbon and with carbon film coating and Sorin mechanical heart valve measurement.

The patient's condition must be known. Laboratory examination include chemically, hematology, PT (Prothrombin Time) and INR (International Normalized Ratio) examinations necessary for valves condition, especially with mechanical valves. International normalized ratio for mitral valve is $2-2.5$ for aortic valve is $1.5-2$ and for double valve replacement is $2.5-3.5$, noticed that aortic valve has the lowest ratio because the velocity in aortic valve is faster than the velocity in mitral valve, so, the prothrombin will be less and is hard to produce. That's mean the supply pressure at aortic valve is $120 \mathrm{~mL} / \mathrm{Hg}$ and at mitral valve is $<10 \mathrm{~mL} / \mathrm{Hg}$. Also, radiation examination and medications given to the patient, especially, the antibiotic and warfarin.

\section{RESULTS AND DISCUSSION}

In this study, the results covered that the 87 patients aged from $10-80$ years old who underwent valves replacement between January 5, 2014 and December 24, 2017.

Number of the cases and heart valve diseases: Figure 13 presented that the variations between number of the cases and heart valve diseases. Where the most common 


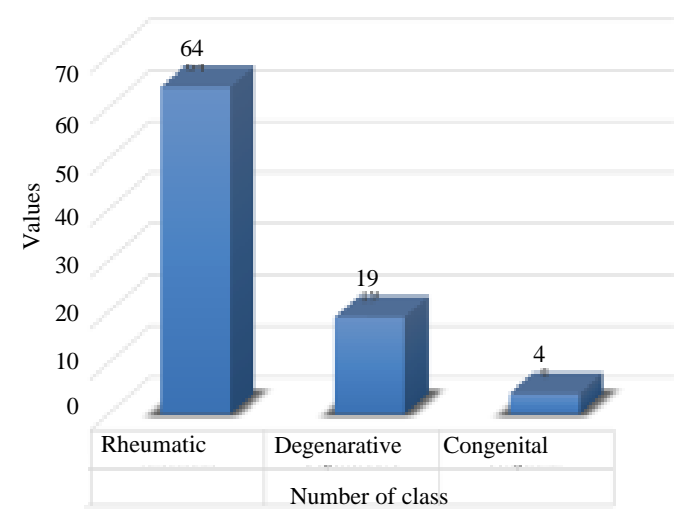

Fig. 13: Variations between number of the cases and heart valve diseases

reasons that cause valve stenosis and valve regurgitation are rheumatic, degenerative and congenital. It can be see that the rheumatic heart disease was the most cause that lead to the heart valves replacement which represented by 64 cases had RHD $73 \%$. While number of the cases for degenerativeand congenital were 19 and 4 cases, respectively. From the results, the highest percentage for cases was 8 through 2016. From the presented results the rheumatic was $73 \%$ but the degenerativeand congenital valve was the most valve has been replaced in 46 cases were 22 and $5 \%$, respectively. This because rheumatic disease is occur because of the streptococcal bacteria that begin in the heart valve and cause valvular damage due to an abnormal immune response to group a streptococcal infection. While degenerative is occur only during age process and that because the body demolition rather than reform, so, the valves are not biology automatically repaired during this lifetime.

Number of the cases and types of valve replacement surgery: Generally, there are three surgeries of heart valve replacement.

Mitral Valve Replacement (MVR): A cardiac surgery that performed when there is a stenosis caused by rheumatic fever and congenital anomalies. In addition to that there is regurgitation because of the rheumatic heart disease, degenerative forms of $\mathrm{MV}$ disease, mitral annular calcification and congenital anomalies. This lead to replace the MV by mechanical or bioprosthetic valve.

Aortic Valve Replacement (AVR): A cardiac surgery performed when the diseased aortic valve replaced by mechanical or bioprosthetic valve when there is stenosis that's caused usually by either degenerative calcification

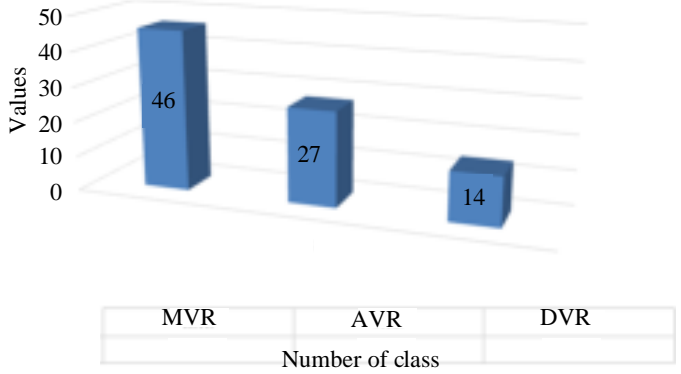

Fig. 14: Variations between number of the cases and types of valve replacement surgery

of trileaflet or caused by progressive stenosis of congenital valve or regurgitation that results from aortic leaflets abnormalities.

Double Valve Replacement (DVR): A cardiac surgical procedure in which a patient's diseased AV and MV are replaced with prosthetic valves. The variation between number of the cases and types of valve replacement surgery are presented in the Fig.14. From 87 cases, mitral (53\%) while aortic valve replaced in 27 cases $(31 \%)$ and the double valve replaced in 14 cases $(16 \%)$. Since, the blood velocity of aortic valve more than the mitral valve, so, percentage of bacteria found less inaortic valve than its percentage in mitral valve also at 40 age the biggestdependency will be on the mitral valve for these reasons $\mathrm{MV}$ is moresusceptible to diseases and damage than AV.

Number of the cases and period time from 2014-2017: Figure 15 presented the number of the cases and period time from January 5, 2014 and December 24, 2017. Number of cases in 2014 was $28(32 \%)$ in 2015 was $26(30 \%)$ in 2016 was $8(9 \%)$ and in 2017 was $25(29 \%)$. The results revealed that the maximum number of the cases was 28 cases through 2014 while the minimum number of the cases was 8 through 2016. From the presented results the number of cases increase through 2014, 2017 while decrease through 2015-2016. The reason of increase and decrease because the number of cases depend on the number of patients who come to the center.

Patients aged from 10-80 years: The results presented in Fig. 16 covered patients aged from $10-80$ years where patients aged between $10-20$ years were $4(4 \%)$ while only $11(13 \%)$ patients between 20-30 had heart valve replacement, $13(15 \%)$ cases between 30-40, 27 (31\%) cases between 40-50. Also, $19(22 \%)$ cases underwent heart valve replacement between 50-60 years old, 11 (13\%) cases between $60-70$ and 2 cases between $60-70$ and 2 (2\%) cases between 70-80. 


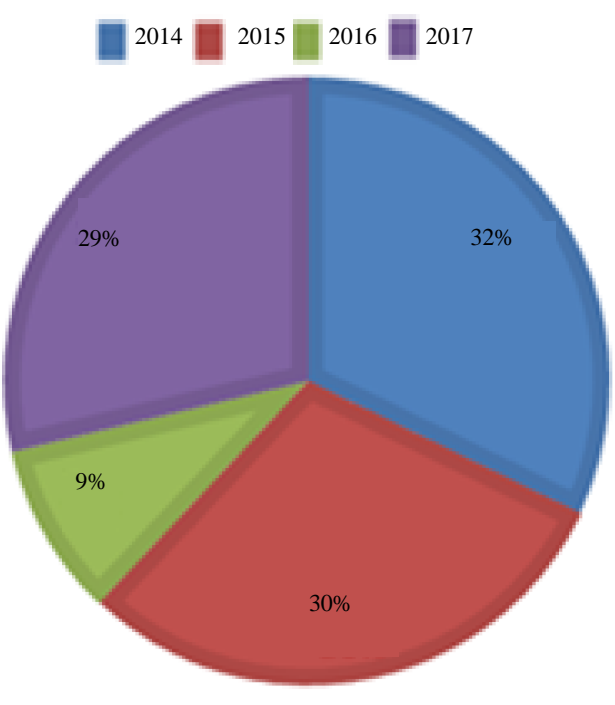

Fig. 15: Number of the cases and period time from 2014- 2017

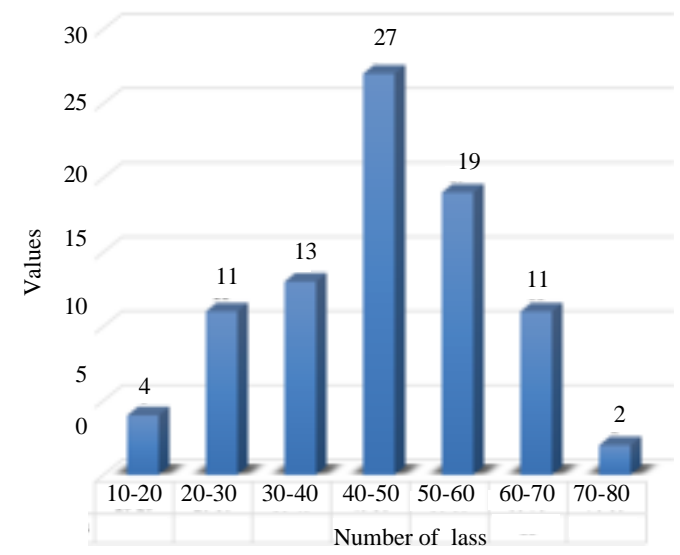

Fig. 16: Variations between number of the cases and their age

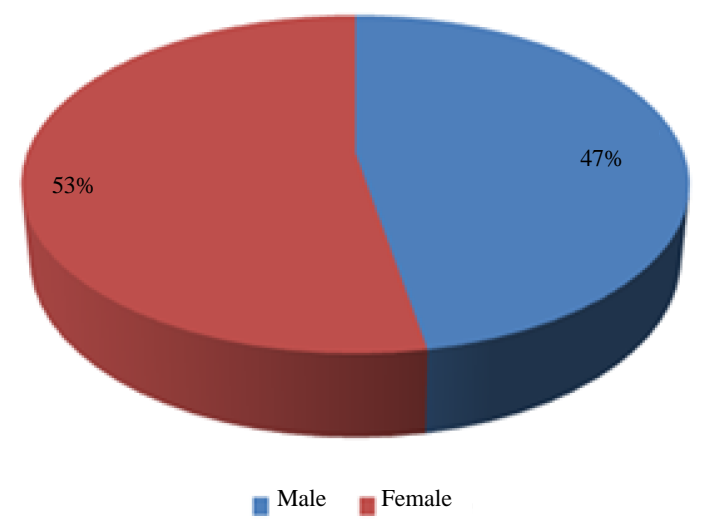

Fig. 17: Illustrated the percentage of cases for both sex who underwent heart valve replacement surgery

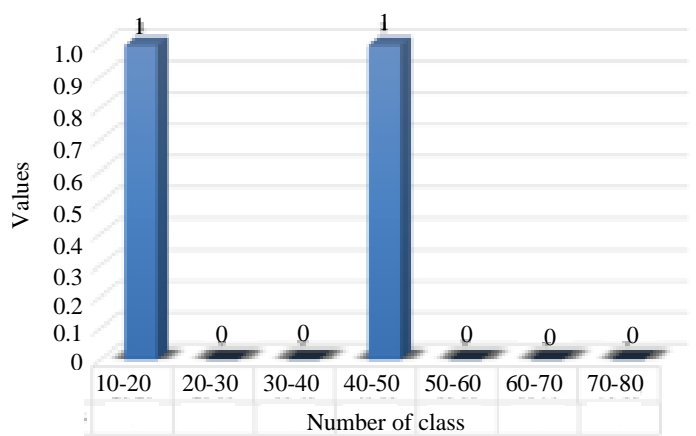

Fig. 18: Number of the deaths for patients who underwent heart valve replacement

The higher number of patients who underwent heart valve replacement was 27 for age between 40-50 years because at this age the valve will be more active and Due to lack of attention and health care to prevent and avoid heart valve causes that will lead to damage and diseases, thus, at the end the damaged heart valve must be replaced. While the lowest number was 2 for aged between $70-80$ years. There were 41 male (47\%) and 46 female (53\%) patients (Fig. 17).

Mortality from 2014-2017: The total center mortality from 2014-2017 presented in Fig. 18 where was just two cases in 26th may. About 2014 patient underwent to MVR surgery at age about 10 years old died because of Deep Vein Thrombosis (DVT). In 17th Feb. 2015 patient underwent to AVR surgery at age 41 years old died because of Cerebro Vascular Accident (CVA).

\section{CONCLUSION}

Rheumatic was the first cause for heart valve replacing by $73 \%$, the main cause for rheumatic is because the streptococcal bacteria that begin in the heart valve and cause valvular damage due to an abnormal immune response to group a streptococcal infection. Acute rheumatic fever which it's the precursor to rheumatic heart disease usually occurs 3 weeks after group A streptococcal pharyngitis and can affect on different organs, the joints, skin, brain and heart and lead to irreversible valve damage and heart failure.

The second cause was the degenerative by $22 \%$, it occurs only during age process and that because the body demolition rather than reform, so, the valves are not biology automatically repaired during this lifetime. Degenerative valve disease is effected mostly on the MV which cause slow degeneration from MV prolapse (improper leaflet movement). Over time the leaflets become 
redundant and floppy and the attachments of the valve will rupture or just thin out. This will cause leakage through the valve. Calcification: the accumulation of calcium on the heart's valves will cause calcification. The aortic valve is most affected than mitral valve. This build-up will make the valve to become harden and thicken which will cause stenosis or narrowing of the aortic valve. This blockage will make the heart to work harder and causing limited in the physical capacities. Calcification is occur due to aging as the calcium amasses in the heart over the lifetime. The lowest percentage was congenital $5 \%$, congenital heart is defecting affect the blood flow through the cardiovascular system. Blood will flow in the wrong direction in abnormal patterns and it will be blocked, partially or completely and that depends on the type of defect that present in the heart. Ranging from mild defects like a malformed valve to the more severe like an absent heart valve, abnormalities of congenital heart require different treatments. In some cases, medicine will be used to treat the condition in others cases, surgery may be necessary. Bicuspid Aortic Valve (BAV) is a defect of the aortic valve that can found present at birth. The aortic valve is allowing oxygen-rich blood to flow from the heart to the aorta. The aorta is the major blood vessel that is bringing blood to the body. Normally, there are three valve flaps (leaflets) found in the aortic valve. In a patient who has a bicuspid aortic valve, there are just only two valve flaps (leaflets) can found in the aortic valve. Valves are the passages for the blood to pass through before leaving each heart chamber. The valves are preventing blood from flowing backwards into the heart. In bicuspid aortic valve, the aortic valve may not be able to stop blood completely from leaking back into the heart (aortic regurgitation). The aortic valve also may become stiff and cannot be open as well, causing the heart to pump harder than usual for passing blood through the valve (aortic stenosis). The ascending aorta also will enlarged (dilated or aneurysm) with this condition. Also, mitral valve replacement was the highest percentage by $53 \%$ due to the blood velocity of aortic valve more than the mitral valve, so, percentage of bacteria found less in aortic valve in contrast to mitral valve for this reason the mitral valve is more susceptible to diseases also at 40 age the biggest dependency will be on the mitral valve for that it is more susceptible to damage.

\section{REFERENCES}

DeWall, R.A., N. Qasim and L. Carr, 2000. Evolution of mechanical heart valves. Ann. Thoracic Surg., 69: 1612-1621.

El Oakley, R., P. Kleine and S.B. David, 2008. Choice of prosthetic heart valve in today's practice. Circulation, 117: 253-256.

Iaizzo, P.A., 2009. Handbook of Cardiac Anatomy Physiology and Devices. 2nd Edn., Springer, Berlin, Germany, ISBN:978-1-60327-371-8,

Maganti, K., V.H. Rigolin, M.E. Sarano and R.O. Bonow, 2010. Valvular heart disease: Diagnosis and management. Mayo Clin. Proc., 85: 483-500.

Morse, D., R.M. Steiner and J. Fernandez, 2012. Guide to Prosthetic Cardiac Valves. Springer, Berlin, Germany, ISBN-13:978-1-4612-9562-4, Pages: 347.

O'Brien, M.F., S. Harrocks, E.G. Stafford, M.A. Gardner and P.G. Pohlner et al., 2001. The homograft aortic valve: A 29-year, 99.3\% follow up of 1,022 valve replacements. J. Heart Valve Dis., 10: 334-344.

Rahimtoola, S.H., 2010. Choice of prosthetic heart valve in adults: Anupdate. J. Am. Coll. Cardiol., 55: 2413-2426.

Snell, R.S., 2000. Clinical Anatomy for Medical Students. 6th Edn., Lippincott Williams \& Wilkins, Philadelphia, Pennsylvania, ISBN-13:9780781715744,

Wang, S.S., J.D. Mph and T.X. O'Brien, 2007. Aortic regurgitation. WebMD LLC., Las Vagas, Nevada, USA. 